\title{
Rheological study of polymer flow past rough surfaces with slip boundary conditions
}

\author{
Anoosheh Niavarani and Nikolai V. Priezjev \\ Department of Mechanical Engineering, \\ Michigan State University, East Lansing, Michigan 48824
}

(Dated: October 30, 2018)

\begin{abstract}
The slip phenomena in thin polymer films confined by either flat or periodically corrugated surfaces are investigated by molecular dynamics and continuum simulations. For atomically flat surfaces and weak wall-fluid interactions, the shear rate dependence of the slip length has a distinct local minimum which is followed by a rapid increase at higher shear rates. For corrugated surfaces with wavelength larger than the radius of gyration of polymer chains, the effective slip length decays monotonically with increasing corrugation amplitude. At small amplitudes, this decay is reproduced accurately by the numerical solution of the Stokes equation with constant and rate-dependent local slip length. When the corrugation wavelength is comparable to the radius of gyration, the continuum predictions overestimate the effective slip length obtained from molecular dynamics simulations. The analysis of the conformational properties indicates that polymer chains tend to stretch in the direction of shear flow above the crests of the wavy surface.

PACS numbers: 83.50.Rp, 83.50.Lh, 68.35.Ct, 61.20.Ja, 36.20.Еy
\end{abstract}

\section{INTRODUCTION}

The dynamics of fluid flow in confined geometries has gained renewed interest due to the recent developments in micro- and nanofluidics [1]. The investigations are motivated by important industrial applications including lubrication, coating, and painting processes. The flow behavior at the sub-micron scale strongly depends on the boundary conditions at the liquid/solid interface. A number of experimental studies on fluid flow past nonwetting surfaces have shown that the conditions at the boundary deviate from the no-slip assumption [2]. The most popular Navier model relates the slip velocity (the relative velocity of the 
fluid with respect to the adjacent solid wall) and the shear rate with the proportionality coefficient, the slip length, which is determined by the linear extrapolation of the fluid velocity profile to zero. The magnitude of the slip length depends on several key parameters, such as wettability [3, 4, 5, 6], surface roughness [7, 8, 9, 10, 11, 12], complex fluid structure [13, 14], and shear rate [15, 16, 17]. However, the experimental determination of the slip length as a function of these parameters is hampered by the presence of several factors with competing effects on the wall slip, e.g. surface roughness and wettability [7] or surface roughness and shear rate [8].

In recent years, molecular dynamics (MD) simulations have been widely used to examine the slip flow past atomically smooth, homogeneous surfaces [18, 19, 20, 21, 22, 23, 24, 25, 26, 27]. The advantage of the MD approach is that the velocity profiles and shear stresses are resolved at the molecular level. The slip length in the shear flow of simple fluids past crystalline walls is a function of the wall-fluid density ratio [19, 21], the relative size of wall atoms and fluid molecules [21, 26], the surface energy [19, 21, 27], and the interfacial shear rate [21, 25, 27]. Weak wall-fluid interactions and incommensurable structures of the solid and fluid phases at the interface usually lead to enhancement of slip [19, 21, 24, 27]. If the slip length at low shear rates is about several molecular diameters then it increases with the shear rate, and the slope of the rate dependence is greater for weaker wall-fluid interactions [21, 27]. The rate dependence of the slip length in the flow of polymer melts is more complicated because of the additional length and time scales associated with the dynamics of polymer chains at the interface and the shear thinning viscosity [20, 25, 28, 29, 30].

In the presence of surface roughness [31, 32, 33, 34, 35] or chemical patterning [36, 37, 38, 39, 40], the fluid flow near the solid boundary is perturbed on the length scales of the surface heterogeneities and its description requires definitions of the effective slip length and the average location of the reference plane. Most commonly, the location of the reference plane is defined as the mean height of the surface asperities, and the shear rate is determined by averaging of the fluid flow over the typical length scale of the surface inhomogeneities. In general, the surface roughness is expected to reduce the effective slip length for wetting liquids [32, 33, 35]. For sufficiently rough surfaces the no-slip boundary condition can be achieved even if the local condition is of zero shear stress [41]. However, in special cases, when the fluid is partially dewetted at the nanostructured, or the so-called "superhydrophobic" surfaces, the slip length might be enhanced up to a few microns [42, 43, 44, 45, 46, 47, 48]. 
In recent MD studies on shear flow of simple fluids, the behavior of the effective slip length was investigated in the Couette cell with either mixed boundary conditions [38] or periodic surface roughness [33]. In the first study, the lower stationary wall with mixed boundary conditions was patterned with a periodic array of stripes representing alternating regions of finite slip and zero shear stress. In the other study [33], the periodically roughened surface was modeled by introducing a sinusoidal offset to the position of the wall atoms. At the wavy wall, the local slip length is modified by the presence of curvature and becomes positiondependent along the curved boundary [49, 50]. A detailed comparison between continuum analysis and MD simulations shows an excellent agreement between the velocity profiles and effective slip lengths when the characteristic length scale of substrate inhomogeneities is larger than approximately thirty molecular diameters [33, 38]. In the case of rough surfaces, an additional correction due to variable wall density was incorporated in the analysis [33]. The problem of applicability of the results obtained for monoatomic fluids to polymer melts is important for modeling polymer flows in confined geometries and design of the hybrid continuum-atomistic algorithms.

In this paper, the MD simulations are carried out to study the dynamic behavior of the slip length at the interface between a polymer melt and atomically flat or periodically corrugated surfaces. The MD results for flat crystalline walls confirm previous findings [30] that the slip length goes through a local minimum at low shear rates and then increases rapidly at higher shear rates. For periodically corrugated surfaces and wetting conditions, the effective slip length decreases gradually with increasing values of the wavenumber. The solution of the Stokes equation with either constant or rate-dependent local slip length is compared with the MD simulations for corrugated surfaces with wavelengths ranging from molecular dimensions to values much larger than the radius of gyration of polymer chains. The orientation and the dynamics of linear polymer chains are significantly affected by surface roughness when the corrugation wavelengths are comparable with the radius of gyration.

The rest of the paper is organized as follows. The details of molecular dynamics and continuum simulations are described in the next section. The dynamic response of the slip length and shear viscosity in the cell with atomically flat surfaces is presented in Sec.III. The results of MD simulations for corrugated walls with large wavelengths and comparison with continuum predictions are reported in Sec.IV. The slip behavior for small wavelengths and 
the conformational properties of the polymer chains near the rough surfaces are analyzed in Sec.V. The summary is given in the last section.

\section{THE DETAILS OF THE NUMERICAL SIMULATIONS}

\section{A. Molecular dynamics model}

The computational domain consists of a polymeric fluid confined between two atomistic walls. Figure1 shows the MD simulation setup. Any two fluid monomers within a cut-off distance of $r_{c}=2.5 \sigma$ interact through the truncated Lennard-Jones (LJ) potential

$$
V_{L J}(r)=4 \varepsilon\left[\left(\frac{\sigma}{r}\right)^{12}-\left(\frac{\sigma}{r}\right)^{6}\right]
$$

where $\varepsilon$ is the energy scale and $\sigma$ is the length scale of the fluid phase. The LJ potential was also employed for the wall-fluid interaction with $\varepsilon_{w f}=\varepsilon$ and $\sigma_{w f}=\sigma$. The wall atoms do not interact with each other, and the wall-fluid parameters are fixed throughout the study. In addition to the LJ potential, the neighboring monomers in a polymer chain $(N=20$ beads) interact with the finite extensible nonlinear elastic (FENE) potential

$$
V_{F E N E}(r)=-\frac{1}{2} k r_{o}^{2} \ln \left[1-\left(\frac{r}{r_{o}}\right)^{2}\right]
$$

where $r_{o}=1.5 \sigma$ and $k=30 \varepsilon \sigma^{-2}[51]$. The MD simulations were performed at a constant density ensemble with $\rho=0.88 \sigma^{-3}$. The total number of fluid monomers is $N_{f}=67200$.

The motion of the fluid monomers was weakly coupled to an external thermal reservoir [52]. To avoid a bias in the flow direction, the random force and the friction term were added to the equation of motion in the $\hat{y}$ direction [19]

$$
m \ddot{y}_{i}=-\sum_{i \neq j} \frac{\partial\left(V_{L J}+V_{F E N E}\right)}{\partial y_{i}}-m \Gamma \dot{y}_{i}+f_{i}(t)
$$

where $\Gamma=1.0 \tau^{-1}$ is a friction constant that regulates the rate of heat flux between the fluid and the heat bath, and $f_{i}(t)$ is the random force with zero mean and variance $2 m \Gamma k_{B} T \delta(t)$ determined from the fluctuation-dissipation theorem [53]. Temperature of the Langevin thermostat is set to $T=1.1 \varepsilon / k_{B}$, where $k_{B}$ is the Boltzmann constant. The equations of motion were integrated using the Verlet algorithm [54] with a time step $\Delta t=0.005 \tau$, where $\tau=\sqrt{m \sigma^{2} / \varepsilon}$ is the characteristic LJ time. 


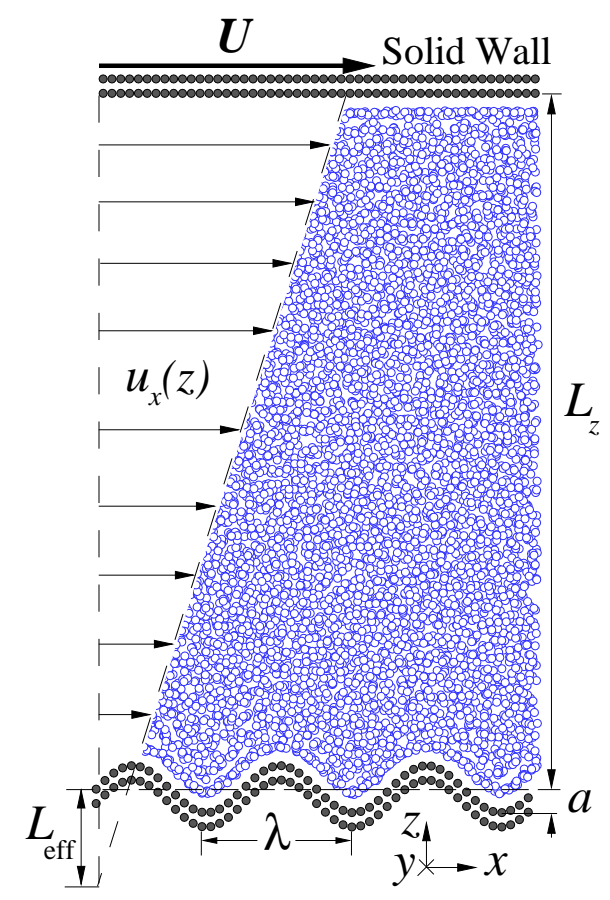

FIG. 1: (Color online) A snapshot of the fluid monomers (open circles) confined between solid walls (closed circles) obtained from the MD simulations. The atoms of the stationary lower wall are distributed along the sinusoidal curve with the wavelength $\lambda$ and amplitude $a$. The flat upper wall is moving with a constant velocity $U$ in the $\hat{x}$ direction. The effective slip length $L_{\text {eff }}$ is determined by the linear extrapolation of the velocity profile to $u_{x}=0$.

The dimensions of the system in the $x y$ plane, unless specified otherwise, were set to $L_{x}=66.60 \sigma$ and $L_{y}=15.59 \sigma$. The upper wall was composed of two layers of an fcc lattice with density $\rho_{w}=1.94 \sigma^{-3}$, which corresponds to the nearest-neighbor distance of $d=0.9 \sigma$ between wall atoms in the (111) plane. The lower wall was constructed of two fcc layers of atoms distributed along the sinusoidal curve with the wavelength $\lambda$ and amplitude $a$. For the largest wavelength $\lambda=66.60 \sigma$, the density of the lower wall $\rho_{w}=1.94 \sigma^{-3}$ was kept uniform along the sinusoid (by including additional rows of atoms parallel to the $\hat{y}$ axis) to avoid additional analysis of the effective slip length due to variable wall density [33]. In the present study, the corrugation amplitude was varied in the range $0 \leqslant a / \sigma \leqslant 12.04$. In the absence of the imposed corrugation $(a=0)$ the distance between the inner fcc planes is set to $L_{z}=74.15 \sigma$ in the $\hat{z}$ direction. Periodic boundary conditions were imposed in the $\hat{x}$ and $\hat{y}$ directions.

The initial velocities of the fluid monomers were chosen from the Maxwell-Boltzmann 
probability distribution at the temperature $T=1.1 \varepsilon / k_{B}$. After an equilibration period of about $3 \times 10^{4} \tau$ with stationary walls, the velocity of the upper wall was gradually increased in the $\hat{x}$ direction from zero to its final value during the next $2 \times 10^{3} \tau$. Then the system was equilibrated for an additional period of $6 \times 10^{3} \tau$ to reach steady-state. Averaging time varied from $10^{5} \tau$ to $2 \times 10^{5} \tau$ for large and small velocities of the upper wall respectively. The velocity profiles were averaged within horizontal slices of $L_{x} \times L_{y} \times \Delta z$, where $\Delta z=$ $0.2 \sigma$. Fluid density profiles near the walls were computed within slices with thickness $\Delta z=0.01 \sigma[27]$.

\section{B. Continuum method}

A solver based on the finite element method was developed for the two-dimensional steady-state and incompressible Navier-Stokes (NS) equation. The NS equation with these assumptions is reduced to

$$
\rho(\mathbf{u} \cdot \nabla \mathbf{u})=-\nabla p+\mu \nabla^{2} \mathbf{u}
$$

where $\mathbf{u}$ is the velocity vector, $\rho$ is the fluid density, and $p$ and $\mu$ are the pressure field and viscosity of the fluid respectively.

The incompressibility condition is satisfied by a divergence-free velocity field $\mathbf{u}$. In order to avoid the decoupling of the velocity and the pressure fields in the numerical simulation of the incompressible flow, the penalty formulation is adopted [55]. This method replaces the continuity equation, $\nabla \cdot \mathbf{u}=0$, with a perturbed equation

$$
\nabla \cdot \mathbf{u}=-\frac{p}{\Lambda}
$$

where $\Lambda$ is the penalty parameter. For most practical applications, where computation is performed with double-precision 64 bit words, a penalty parameter between $10^{7}$ and $10^{9}$ is sufficient to conserve the accuracy [55]. In our simulations the incompressibility constraint was set to $\Lambda=10^{7}$. The pressure term in Eq. (5) is then substituted into the NS equation. The equation Eq. (4) can be rewritten as follows:

$$
\rho(\mathbf{u} \cdot \nabla \mathbf{u})=\Lambda \nabla(\nabla \cdot \mathbf{u})+\mu \nabla^{2} \mathbf{u}
$$

where the continuity equation is no longer necessary [55]. The NS equation is integrated with the Galerkin method using bilinear rectangular isoparametric elements [55]. 
Four boundary conditions must be specified for the continuum simulation. Periodic boundary conditions are used for the inlet and outlet along the $\hat{x}$ direction. A slip boundary condition is applied at the upper and lower walls. In the local coordinate system (spanned by the tangential vector $\vec{t}$ and the normal vector $\vec{n}$ ), the fluid velocity along the curved boundary is calculated as

$$
u_{t}=L_{0}\left[(\vec{n} \cdot \nabla) u_{t}+u_{t} / R\right]
$$

where $u_{t}$ is the tangential component of $\mathbf{u}=u_{t} \vec{t}+u_{n} \vec{n}, L_{0}$ is the slip length at the flat liquid/solid interface, the term in the brackets is the local shear rate, and $R$ is the local radius of curvature [50]. The radius of curvature is positive for the concave and negative for the convex regions. For a flat surface, $R \rightarrow \infty$, the boundary condition given by Eq. (7) simply becomes the Navier slip law.

The simulation is started by applying the no-slip boundary condition as the initial guess. Once the equations of motion are solved implicitly, the local slip velocities at the lower and upper boundaries are updated using Eq. (7). In the next step, the equations of motion are solved with the updated slip velocities used as a new boundary condition. The iterative procedure is repeated until the solution converges to a desired accuracy. The convergence rate of the iterative solution remains under control with the under-relaxation value about 0.001 for the boundary nodes. In all continuum simulations, the grids at the lower boundary have an aspect ratio of about one. The computational cost is reduced by increasing the aspect ratio of the grids in the bulk region.

The normalized average error value in the simulation is defined as

$$
\text { error }=\left[\sum_{i=1}^{N_{p}} \frac{\left|\mathbf{u}_{i}^{n}-\mathbf{u}_{i}^{n+1}\right|}{\left|\mathbf{u}_{i}^{n+1}\right|}\right] / N_{p}
$$

where $N_{p}$ is the number of nodes in the system, $\mathbf{u}_{i}^{n}$ is the velocity at the node $i$ and time step $n$, and $\mathbf{u}_{i}^{n+1}$ is the velocity in the next time step. The typical error in the converged solution is less than $10^{-9}$. At the boundaries the solution satisfies $u_{t}=L_{\text {local }} \frac{\partial u_{t}}{\partial n}$, where the local slip length is $L_{\text {local }}=\left(L_{0}^{-1}-R^{-1}\right)^{-1}[\underline{50}]$.

\section{MD RESULTS FOR FLAT WALLS}

The averaged velocity profiles for selected values of the upper wall speed $U$ are presented in Fig.2. The profiles are linear throughout the cell, except for $U \geqslant 6.0 \sigma / \tau$ where a slight 


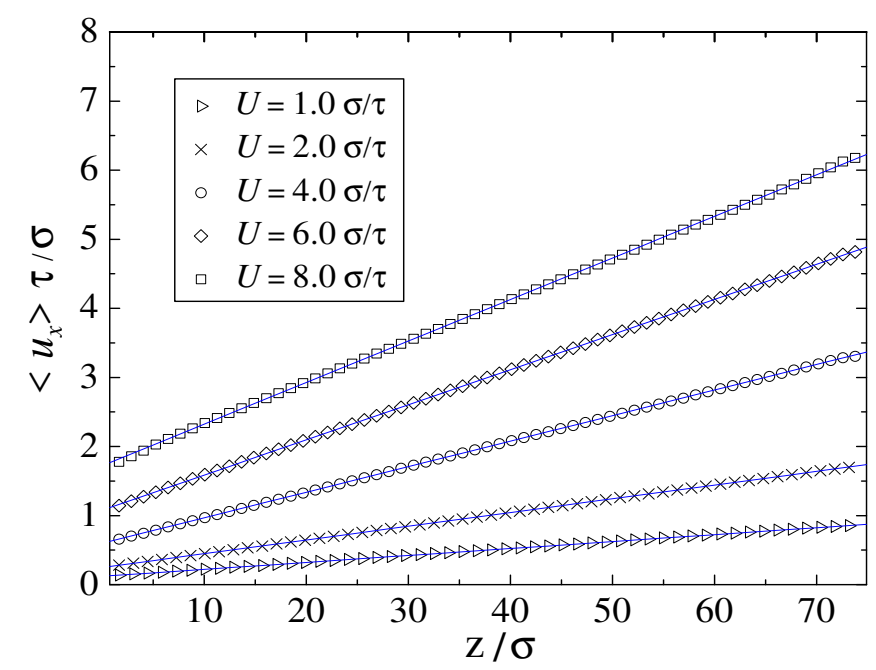

FIG. 2: (Color online) Averaged velocity profiles in the cell with flat upper and lower walls. The solid lines are the best linear fit to the data. The vertical axes indicate the location of the fcc lattice planes. The velocities of the upper wall are tabulated in the inset.

curvature appears in the region of about $4 \sigma$ near the walls. Note that the relative slip velocity at the upper and lower walls increases with increasing upper wall speed. The shear rate was determined from the linear fit to the velocity profiles across the whole width of the channel (see Fig.2). The uncertainty in the estimated value of shear rate is due to the thermal fluctuations and the slight curvature in the velocity profiles near the walls. The typical error bars for the shear rate are about $2 \times 10^{-5} \tau^{-1}$ and $6 \times 10^{-4} \tau^{-1}$ for small and large upper wall speeds respectively (not shown).

In this study, the shear stress in steady-state flow was computed from the Irving-Kirkwood relation [56]. The dynamic response of the fluid viscosity with increasing shear rate is presented in Fig.3. At higher shear rates, the fluid exhibits shear thinning behavior with the slope of about -0.33 . Although the power law coefficient is larger than the reported values in experimental studies [57], the results are consistent with previous MD simulations of polymer melts for similar flow conditions [30, 58, 59].

The slip length was calculated by the linear extrapolation of the fluid velocity profile to zero with respect to a reference plane, which is located $0.5 \sigma$ away from the fcc lattice plane [27, 30]. Figure 4 shows the rate dependence of the slip length in the same range of shear rates as in Fig. 3. The slip length goes through a shallow minimum at low shear rates and then increases rapidly at higher rates. The error bars are larger at low shear rates 


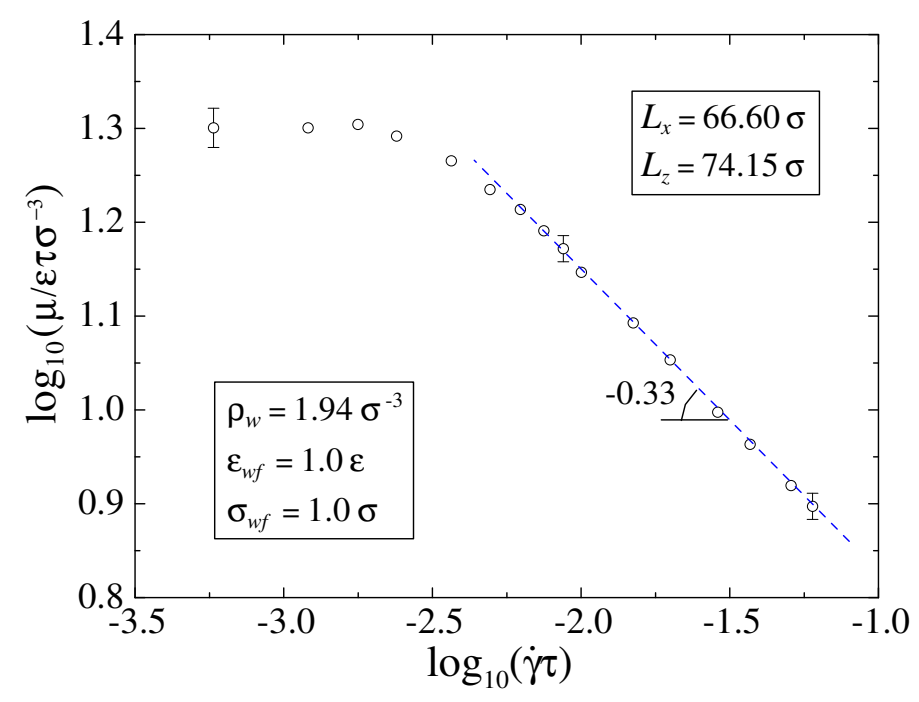

FIG. 3: (Color online) Viscosity of the polymer melt $\mu / \varepsilon \tau \sigma^{-3}$ as a function of shear rate. The dashed line with the slope -0.33 is plotted for reference.

because the thermal fluid velocity $v_{T}^{2}=k_{B} T / m$ is greater than the average flow velocity. The nonmonotonic behavior of the slip length in sheared polymer films with atomically flat surfaces can be interpreted in terms of the friction coefficient at the liquid/solid interface, which undergoes a gradual transition from a nearly constant value to the power law decay as a function of the slip velocity [30]. The data for the slip length shown in Fig.[4 are well fitted by the fourth-order polynomial

$$
L_{0}(x) / \sigma=16.8-72.0 \times 10 x+44.0 \times 10^{3} x^{2}-97.3 \times 10^{4} x^{3}+80.5 \times 10^{5} x^{4},
$$

where $x=\dot{\gamma} \tau$ is the shear rate. The polynomial fit will be used to specify the boundary conditions for the continuum solution described in the next section.

\section{RESULTS FOR PERIODICALLY CORRUGATED WALLS: LARGE WAVELENGTH}

\section{A. MD simulations}

The monomer density profiles were computed in the averaging regions located in the grooves and above the peaks of the corrugated lower wall with wavelength $\lambda=L_{z}=66.60 \sigma$. The dimensions of the averaging regions are set to $0.5 \sigma$ and $L_{y}=15.59 \sigma$ in the $\hat{x}$ and $\hat{y}$ directions respectively. Figure 5 shows the monomer density profiles near the upper and 


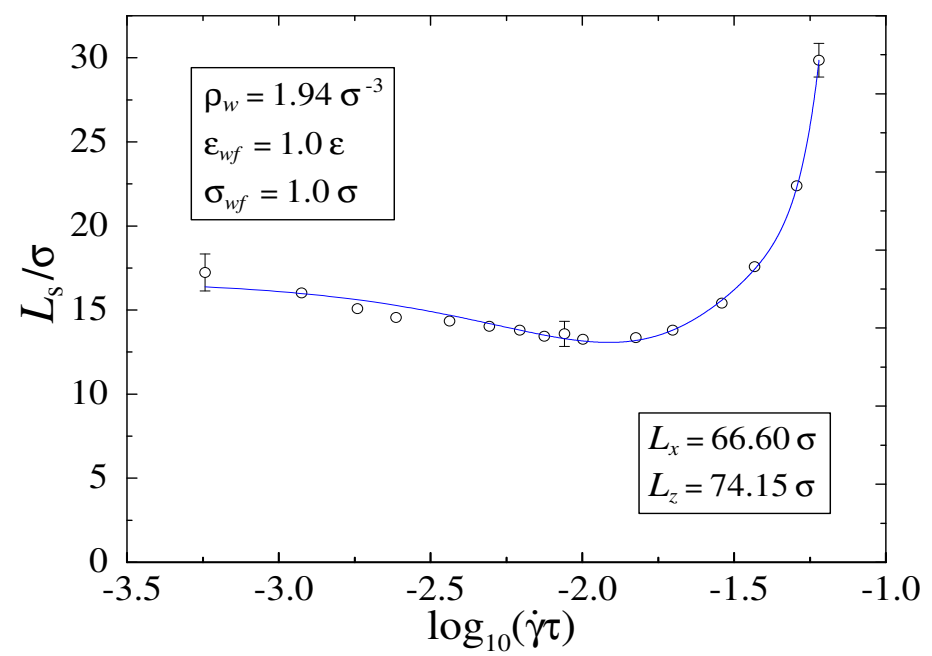

FIG. 4: (Color online) Variation of the slip length as a function of shear rate in the cell with flat upper and lower walls. The solid line is a fourth-order polynomial fit to the data given by Eq. (9).

lower walls at equilibrium. The pronounced density oscillations are attributed to the successive layering of the fluid monomers near the walls. These oscillations decay within a few molecular diameters from the walls to a uniform profile characterized by the bulk density of $\rho=0.88 \sigma^{-3}$. Note that the height of the first peak in the density profile inside the groove is slightly larger than its value above the crest [see Fig.[5(b)]. The fluid monomers experience stronger net surface potential in the groove than above the crest because of the closer spatial arrangement of the wall atoms around the location of the first density peak in the groove. This effect is amplified when the local radius of curvature at the bottom of the grooves is reduced at smaller wavelengths (see below).

The averaged velocity profiles in the cell with periodically corrugated lower and flat upper walls are presented in Fig.6. The fluid velocity profiles were averaged within horizontal slices of thickness $\Delta z=0.2 \sigma$ distributed uniformly from the bottom of the grooves to the upper wall. With increasing corrugation amplitude, the slip velocity decreases and the profiles acquire a curvature near the lower wall. Note also that the relative slip velocity at the upper wall increases with the shear rate. The linear part of the velocity profiles, $30 \leqslant z / \sigma \leqslant 60$, was used to determine the effective slip length $L_{\text {eff }}$ at the corrugated lower wall. The variation of the effective slip length as a function of wavenumber $k a=2 \pi a / \lambda$ is shown in Fig. 7 . The slip length decreases monotonically with increasing wavenumber and becomes negative at $k a \gtrsim 0.7$. The results of comparison between the MD and continuum simulations for three 


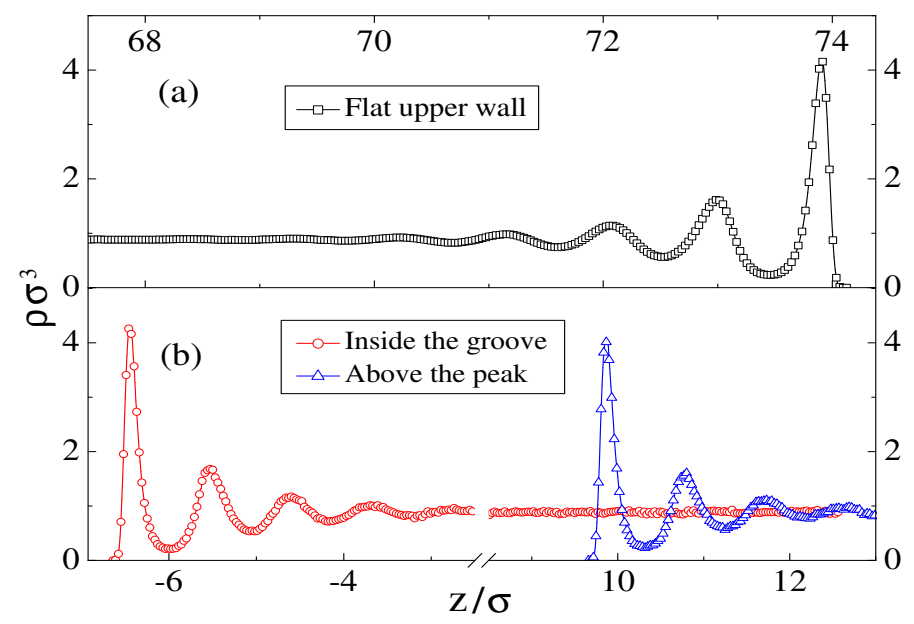

FIG. 5: (Color online) Averaged density profiles near the stationary upper wall (a), above the peak and in the groove of the lower wall with amplitude $a=8.16 \sigma$ and wavelength $\lambda=66.60 \sigma$ (b).

different cases are presented in the next section.

\section{B. Comparison between MD and continuum simulations}

In continuum simulations, the length, time and energy scales are normalized by the LJ parameters $\sigma, \tau$ and $\varepsilon$ respectively. The continuum nondimensional parameters are denoted by the $\left({ }^{\sim}\right)$ sign. The size of the two-dimensional domain is fixed to $66.60 \times 73.15$ in the $\hat{x}$ and $\hat{z}$ directions, respectively. The following three cases were examined: the Stokes solution with constant slip length in Eq. (7), the Stokes solution with shear-rate-dependent slip length given by Eq. (9), and the Navier-Stokes solution with constant slip length in Eq. (17). For all

cases considered, the flat upper wall is translated with a constant velocity $\tilde{U}=0.5$ in the $\hat{x}$ direction. Similarly to the MD method, the effective slip length is defined as a distance from the reference plane at $a=0$ to the point where the linearly extrapolated velocity profile vanishes.

In the first case, the finite element method was implemented to solve the Stokes equation with boundary conditions at the upper and lower walls specified by Eq. (7) with $\tilde{L}_{0}=14.1$. This value corresponds to the slip length $L_{0}=14.1 \pm 0.5 \sigma$ extracted from the MD simulations in the cell with flat walls and the velocity of the upper wall $U=0.5 \sigma / \tau$. Note that at large amplitudes $\tilde{a} \geqslant 8.16$, the normal derivative of the tangential velocity $\partial u_{t} / \partial n$ at the bottom of the grooves is negative, while the $\hat{x}$ component of the slip velocity is positive 


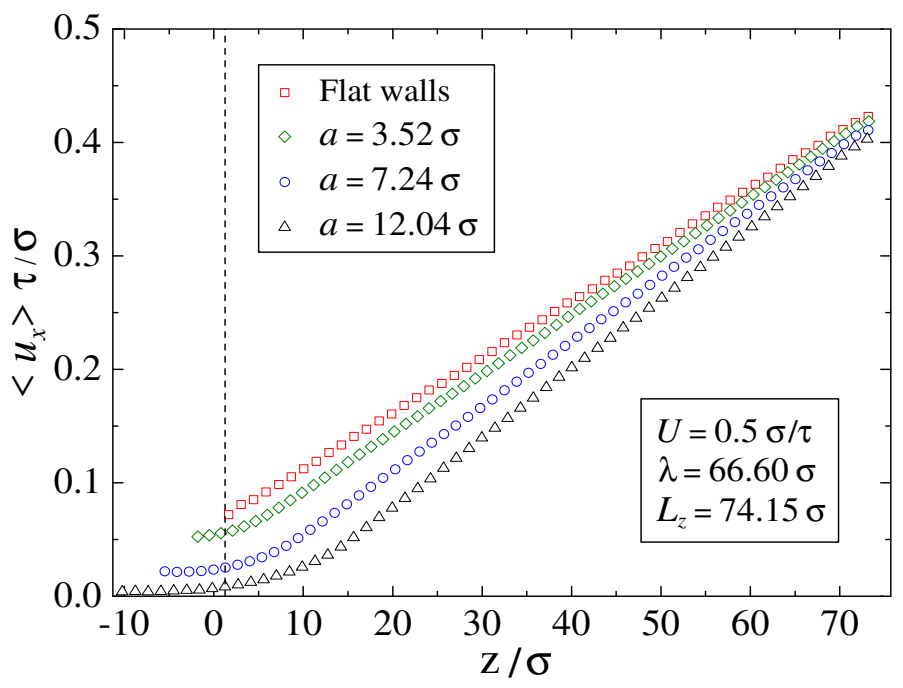

FIG. 6: Averaged velocity profiles for the indicated values of the corrugation amplitude $a$. The vertical dashed line denotes a reference plane for calculation of the effective slip length at the corrugated lower wall. The velocity of the flat upper wall is $U=0.5 \sigma / \tau$.

everywhere along the corrugated lower wall. The dependence of the effective slip length on the corrugation amplitude is shown in Fig.7. The continuum results agree well with the approximate analytical solution [50] for $k a \lesssim 0.5$ (not shown). For larger amplitudes, $k a>$ 0.5, where the analytical solution is not valid, our results were tested to be grid independent. There is an excellent agreement between slip lengths obtained from the MD and continuum simulations for $k a \lesssim 0.3$. With further increasing the amplitude, the slip length obtained from the continuum solution overestimates its MD value. The results presented in Fig.7 are consistent with the analysis performed earlier for simple fluids [33], although a better agreement between MD and continuum solutions was expected at $k a>0.5$ because of the larger system size considered in the present study.

As discussed in the previous section, the slip length for atomically flat walls is ratedependent even at low shear rates (see Fig.44). In the second case, we include the effect of shear rate in the analysis of the effective slip length at the corrugated lower wall and flat upper wall. The Stokes equation is solved with boundary conditions given by Eq. (17), where the slip length Eq. (9) is a function of the local shear rate at the curved and flat boundaries. The results obtained from the Stokes solution with constant and rate-dependent slip lengths are almost indistinguishable (see Fig.77). This behavior can be attributed to a small variation of the intrinsic slip length Eq. (9) at low shear rates. For example, at the largest amplitude, 


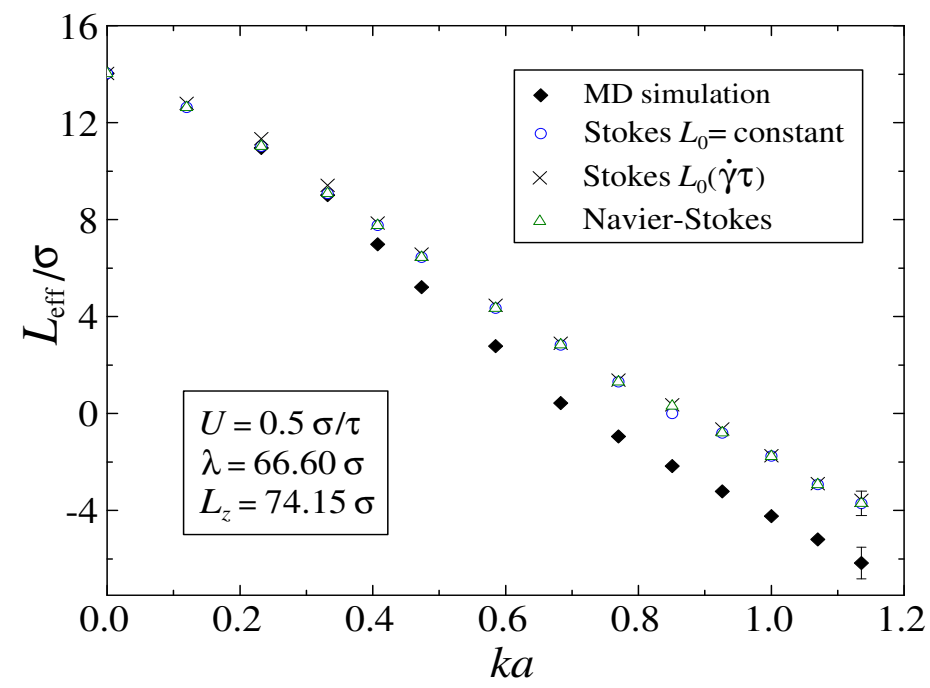

FIG. 7: The effective slip length as a function of wavenumber $k a$ obtained from the MD simulations $(\checkmark)$, the solution of the Stokes equation with rate-independent slip length $L_{0}(\circ)$ and with $L_{0}(\dot{\gamma} \tau)$ given by Eq. (9) $(\times)$, the solution of the Navier-Stokes equation with $L_{0}(\triangle)$.

$\tilde{a}=12.04$, the local shear rate at the corrugated wall is position-dependent and bounded by $\left|\partial u_{t} / \partial n+u_{t} / R\right| \leqslant 0.0035$. In this range of shear rates, the normalized value of the slip length in Eq. (9) varies between $14.7 \leqslant \tilde{L}_{0} \leqslant 16.6$. It is expected, however, that the effect of shear rate will be noticeable at larger values of the top wall speed $\tilde{U}$.

In the third case, the Navier-Stokes equation is solved with a constant slip length $\tilde{L}_{0}=14.1$ in Eq. (7) at the flat upper and corrugated lower walls. The upper estimate of the Reynolds number based on the fluid density $\tilde{\rho}=0.88$, viscosity $\tilde{\mu}=20.0$, and the fluid velocity difference across the channel is $R e \approx 1.3$. It was previously shown by Tuck and Kouzoubov [61] that at small $k a$ and $\tilde{L}_{0}=0$ the magnitude of the apparent slip velocity at the mean surface increases due to finite Reynolds number effects for $R e \gtrsim 30$. In our study, the difference between the slip lengths extracted from the Stokes and Navier-Stokes solutions is within the error bars (see Fig.7). These results confirm that the slip length is not affected by the inertia term in the Navier-Stokes equation for $R e \lesssim 1.3$. To check how sensitive the boundary conditions are to higher Reynolds number flows, we have also repeated the continuum simulations for larger velocity of the upper wall $\tilde{U}=50$, which corresponds to $R e \approx 130$. For the largest corrugation amplitude $\tilde{a}=12.04$, the backflow appears inside the groove and the effective slip length becomes smaller than its value for $\tilde{U}=0.5$ by about 0.7 (not shown). 


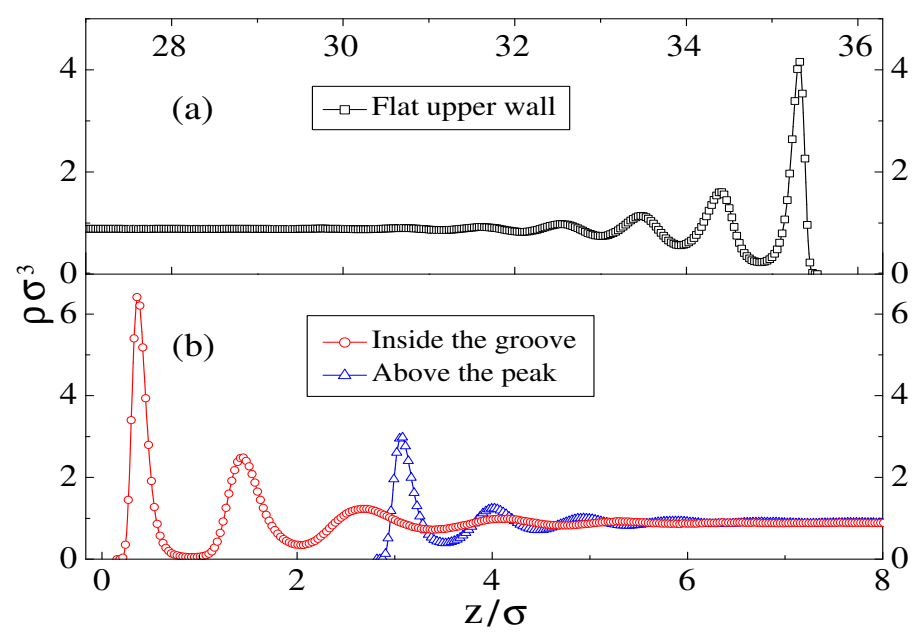

FIG. 8: Averaged fluid density profiles near flat upper wall (a) and corrugated lower wall with amplitude $a=1.4 \sigma$ and wavelength $\lambda=7.5 \sigma(\mathrm{b})$. The velocity of the upper wall is $U=0.5 \sigma / \tau$.

\section{RESULTS FOR PERIODICALLY CORRUGATED WALLS: SMALL WAVELENGTHS}

\section{A. Comparison between MD and continuum simulations}

The MD simulations described in this section were performed at corrugation wavelengths $(\lambda / \sigma=3.75,7.5$ and 22.5) comparable with the size of a polymer coil. Periodic surface roughness of the lower wall was created by displacing the fcc wall atoms by $\Delta z=a \sin (2 \pi x / \lambda)$ in the $\hat{z}$ direction [33]. In order to reduce the computational time the system size was restricted to $N_{f}=8580$ fluid monomers and $L_{x}=22.5 \sigma, L_{y}=12.5 \sigma$ and $L_{z}=35.6 \sigma$. All other system parameters were kept the same as in the previous section.

The representative density profiles near the upper and lower walls are shown in Fig. 8 for the wavelength $\lambda=7.5 \sigma$. The height of the first peak in the density profile is larger in the grooves than near the flat wall or above the crests of the corrugated surface. The effective slip length as a function of wavenumber $k a$ is plotted in Fig.9. For all wavelengths, the slip length decreases monotonically with increasing values of $k a$. At the smallest wavelength $\lambda=3.75 \sigma$, the slip length rapidly decays to zero at $k a \approx 0.4$ and weakly depends on the corrugation amplitude at larger $k a$. Inspection of the local velocity profiles for $\lambda=3.75 \sigma$ and $k a \gtrsim 1.0$ indicates that the flow is stagnant inside the grooves 60 ].

In the continuum analysis, the Stokes equation with a constant slip length $\tilde{L}_{0}=14.1$ in 


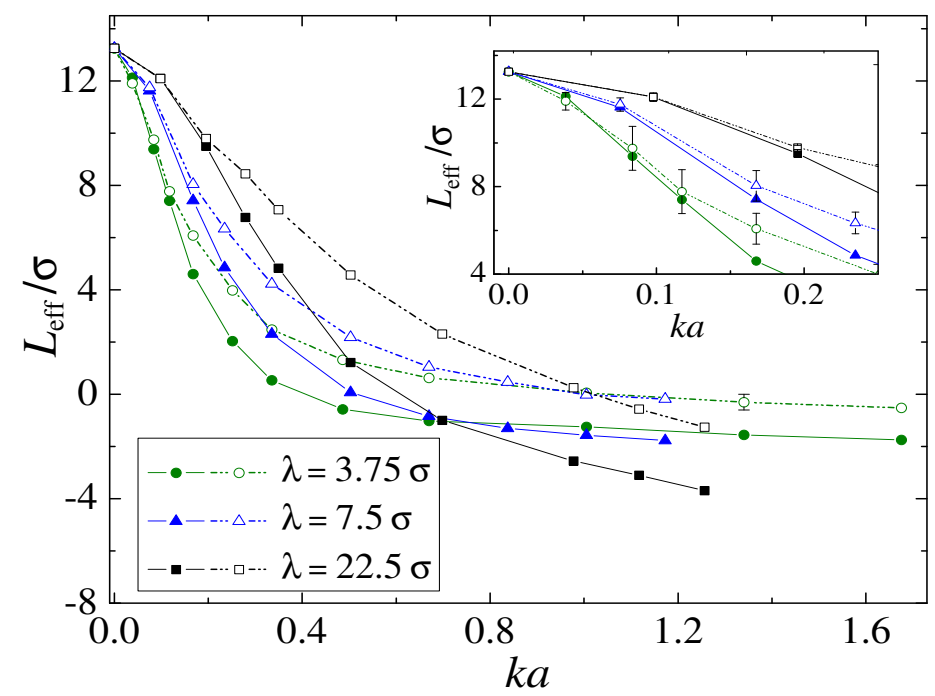

FIG. 9: The effective slip length as a function of wavenumber $k a$ for the indicated values of wavelength $\lambda$. Continuum results are denoted by the dashed lines and open symbols, while the MD results are shown by straight lines and filled symbols. The inset shows the same data for $k a \leqslant 0.25$.

Eq. (17) is solved for the three wavelengths. The comparison between the MD results and the solution of the Stokes equation is presented in Fig.9, The error bars are larger for the smallest wavelength because of the fine grid resolution required near the lower boundary at $k a \lesssim 0.2$ (see inset in Fig.99). The results shown in Fig.9 confirm previous findings for simple fluids [33] that the slip length obtained from the Stokes flow solution overestimates its MD value and the agreement between the two solutions becomes worse at smaller wavelengths. It is interesting to note that the curves for different wavelengths intersect each other at $k a \approx 0.63$ in the MD model and at $k a \approx 1.02$ in the continuum analysis. The same trend was also observed in the previous study on slip flow of simple fluids past periodically corrugated surfaces [33].

\section{B. The polymer chain configuration and dynamics near rough surfaces}

In this section, the properties of polymer chains are examined in the bulk and near the corrugated boundary with wavelengths $\lambda=3.5 \sigma$ and $\lambda=7.5 \sigma$. The radius of gyration $R_{g}$ was computed as

$$
R_{g}^{2}=\frac{1}{N} \sum_{i=1}^{N}\left(R_{i}-R_{c m}\right)^{2}
$$




\begin{tabular}{|c|c|c|c|c|c|c|c|c|c|}
\hline \multirow[t]{2}{*}{$\lambda=7.5 \sigma$} & \multicolumn{3}{|c|}{$a=0.2 \sigma$} & \multicolumn{3}{|c|}{$a=0.6 \sigma$} & \multicolumn{3}{|c|}{$a=1.4 \sigma$} \\
\hline & $R_{g x}$ & $R_{g y}$ & $R_{g z}$ & $R_{g x}$ & $R_{g y}$ & $R_{g z}$ & $R_{g x}$ & $R_{g y}$ & $R_{g z}$ \\
\hline Bulk Equilibrium & 1.18 & 1.18 & 1.18 & 1.18 & 1.18 & 1.18 & 1.18 & 1.18 & 1.18 \\
\hline Shear flow & 1.70 & 1.11 & 1.04 & 1.76 & 1.10 & 1.02 & 1.79 & 1.10 & 1.01 \\
\hline \multirow{2}{*}{$\begin{array}{cc}\text { Upper } & \text { Equilibrium } \\
\text { wall } & \text { Shear flow }\end{array}$} & 1.34 & 1.37 & 0.66 & 1.35 & 1.36 & 0.66 & 1.35 & 1.35 & 0.66 \\
\hline & 1.63 & 1.29 & 0.63 & 1.64 & 1.29 & 0.63 & 1.67 & 1.29 & 0.63 \\
\hline Peak Equilibrium & 1.35 & 1.36 & 0.69 & 1.40 & 1.31 & 0.76 & 1.46 & 1.27 & 0.84 \\
\hline Shear flow & 1.65 & 1.28 & 0.67 & 1.85 & 1.21 & 0.75 & 2.17 & 1.10 & 0.93 \\
\hline \multirow{2}{*}{$\begin{array}{r}\text { Groove Equilibrium } \\
\text { Shear flow }\end{array}$} & 1.31 & 1.37 & 0.63 & 1.10 & 1.47 & 0.61 & 0.77 & 1.69 & 0.61 \\
\hline & 1.50 & 1.34 & 0.62 & 1.19 & 1.44 & 0.60 & 0.75 & 1.89 & 0.59 \\
\hline
\end{tabular}

TABLE I: Averaged $\hat{x}, \hat{y}$, and $\hat{z}$ components of the radius of gyration at equilibrium and in the shear flow. The $R_{g}$ values are reported in the bulk, near the flat upper wall, above the peaks, and inside the grooves. The wavelength of the lower wall is $\lambda=7.5 \sigma$. The size of the averaging region inside the grooves and above the peaks is $\sigma \times 12.5 \sigma \times 1.5 \sigma$. The estimate of the error bars is $\pm 0.03 \sigma$.

where $R_{i}$ is the three-dimensional position vector of a monomer, $N=20$ is the number of monomers in the chain, and $R_{c m}$ is center of mass vector defined as

$$
R_{c m}=\frac{1}{N} \sum_{i=1}^{N} R_{i} .
$$

The chain statistics were collected in four different regions at equilibrium $(U=0)$ and in the shear flow induced by the upper wall moving with velocity $U=0.5 \sigma / \tau$ in the $\hat{x}$ direction. Averaging regions were located above the peaks, in the grooves, near the flat upper wall and in the bulk (see Fig.10 for an example). The dimensions of the averaging regions above the peaks and in the grooves of the lower wall are $\sigma \times 12.5 \sigma \times 1.5 \sigma$, and near the upper wall and in the bulk are $22.5 \sigma \times 12.5 \sigma \times 1.5 \sigma$. Three components of the radius of gyration were computed for polymer chains with the center of mass inside the averaging regions.

In the bulk region, the components of the radius of gyration remain the same for both wavelengths, indicating that the chain orientation is isotropic at equilibrium and is not affected by the confining walls. In the steady-state flow, the effective slip length is suppressed by the surface roughness and, therefore, the shear rate in the bulk increases with the corru- 


\begin{tabular}{|c|c|c|c|c|c|c|c|c|c|}
\hline \multirow{2}{*}{$\lambda=3.75 \sigma$} & \multicolumn{3}{|c|}{$a=0.07 \sigma$} & \multicolumn{3}{c|}{$a=0.2 \sigma$} & \multicolumn{3}{c|}{$a=1.0 \sigma$} \\
\cline { 2 - 11 } & $R_{g x}$ & $R_{g y}$ & $R_{g z}$ & $R_{g x}$ & $R_{g y}$ & $R_{g z}$ & $R_{g x}$ & $R_{g y}$ & $R_{g z}$ \\
\hline \hline Bulk Equilibrium & 1.18 & 1.18 & 1.18 & 1.18 & 1.18 & 1.18 & 1.18 & 1.18 & 1.18 \\
Shear flow & 1.70 & 1.12 & 1.03 & 1.76 & 1.10 & 1.02 & 1.78 & 1.10 & 1.01 \\
\hline Upper Equilibrium & 1.33 & 1.36 & 0.66 & 1.36 & 1.34 & 0.66 & 1.37 & 1.34 & 0.65 \\
wall Shear flow & 1.60 & 1.32 & 0.63 & 1.64 & 1.29 & 0.63 & 1.65 & 1.27 & 0.63 \\
\hline Peak Equilibrium & 1.38 & 1.33 & 0.67 & 1.42 & 1.30 & 0.71 & 1.41 & 1.25 & 0.92 \\
Shear flow & 1.61 & 1.27 & 0.65 & 1.66 & 1.26 & 0.69 & 1.78 & 1.17 & 0.84 \\
\hline Groove Equilibrium & 1.33 & 1.36 & 0.64 & 1.25 & 1.42 & 0.61 & 0.48 & 2.64 & 0.55 \\
Shear flow & 1.60 & 1.29 & 0.61 & 1.60 & 1.33 & 0.59 & 0.47 & 2.70 & 0.54 \\
\hline
\end{tabular}

TABLE II: Averaged $\hat{x}, \hat{y}$, and $\hat{z}$ components of the radius of gyration at equilibrium and in the shear flow. The $R_{g}$ values are reported in the bulk, near the flat upper wall, above the peaks, and inside the grooves. The wavelength of the lower wall is $\lambda=3.75 \sigma$. The dimensions of the averaging region are the same as in Table!.

gation amplitude. This explains why the $\hat{x}$ component of the radius of gyration $R_{g x}$ increases slightly at larger amplitudes (see Tables! and 【I). Near the upper wall, the polymer chains become flattened parallel to the surface and slightly stretched in the presence of shear flow. These results are consistent with the previous MD simulations of polymer melts confined between atomically flat walls [28, 62, 63].

In the case of a rough surface with the wavelength $\lambda=7.5 \sigma$, a polymer chain can be accommodated inside a groove (see Fig.10 for an example). With increasing corrugation amplitude, the polymer chains inside the grooves elongate along the $\hat{y}$ direction and contract in the $\hat{x}$ direction (see Table【). The tendency of the trapped molecules to orient parallel to the grooves was observed previously in MD simulations of hexadecane [32]. In the presence of shear flow, polymer chains are highly stretched in the $\hat{x}$ direction above the crests of the wavy wall. A snapshot of the unfolded chains during migration between neighboring valleys is shown in Fig.10, The flow conditions in Fig.10 correspond to a negative effective slip length $L_{\text {eff }} \approx-2 \sigma$.

For the smallest corrugation wavelength $\lambda=3.75 \sigma$, polymer chains cannot easily fit in the grooves unless highly stretched. Therefore, the $\hat{y}$ component of the radius of gyration 


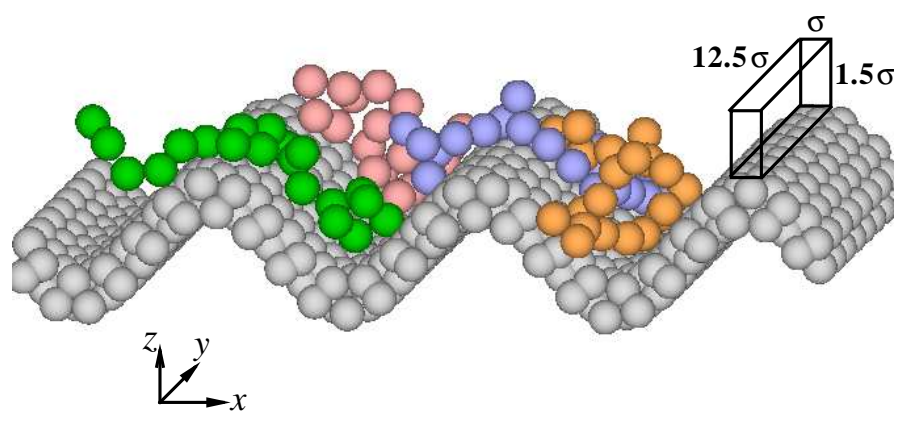

FIG. 10: (Color online) A snapshot of four polymer chains near the lower corrugated wall for wavelength $\lambda=7.5 \sigma$ and amplitude $a=1.4 \sigma$. The velocity of the upper wall is $U=0.5 \sigma / \tau$.

is relatively large when the center of mass is located in the deep grooves (see Table II). Figure11 shows a snapshot of several polymer chains in contact with the lower corrugated wall. The chain segments are oriented parallel to the grooves and stretched above the crests of the surface corrugation. Visual inspection of the consecutive snapshots reveals that the chains near the corrugated wall move, on average, in the direction of shear flow; however, their tails can be trapped for a long time because of the strong net surface potential inside the grooves. For large wavenumbers $k a \gtrsim 0.5$, the magnitude of the negative effective slip length is approximately equal to the sum of the corrugation amplitude and $R_{g z}$ of the polymer chains above the crests of the wavy wall.

\section{CONCLUSIONS}

In this paper the effects of the shear rate and surface roughness on slip flow of a polymer melt was studied using molecular dynamics and continuum simulations. The linear part of the velocity profiles in the steady-state flow was used to calculate the effective slip length and shear rate. For atomically flat walls, the slip length passes through a shallow minimum at low shear rates and then increases rapidly at higher shear rates. In the case of periodic surface heterogeneities with the wavelength larger than the radius of gyration, the effective slip length decays monotonically with increasing the corrugation amplitude. For small wavenumbers, the effective slip length obtained from the solution of the Stokes equation with constant and shear-dependent local slip length is in a good agreement with its values computed from the MD simulations, in accordance with the previous analysis for simple fluids [33]. At low Reynolds numbers, the inertial effects on slip boundary conditions 


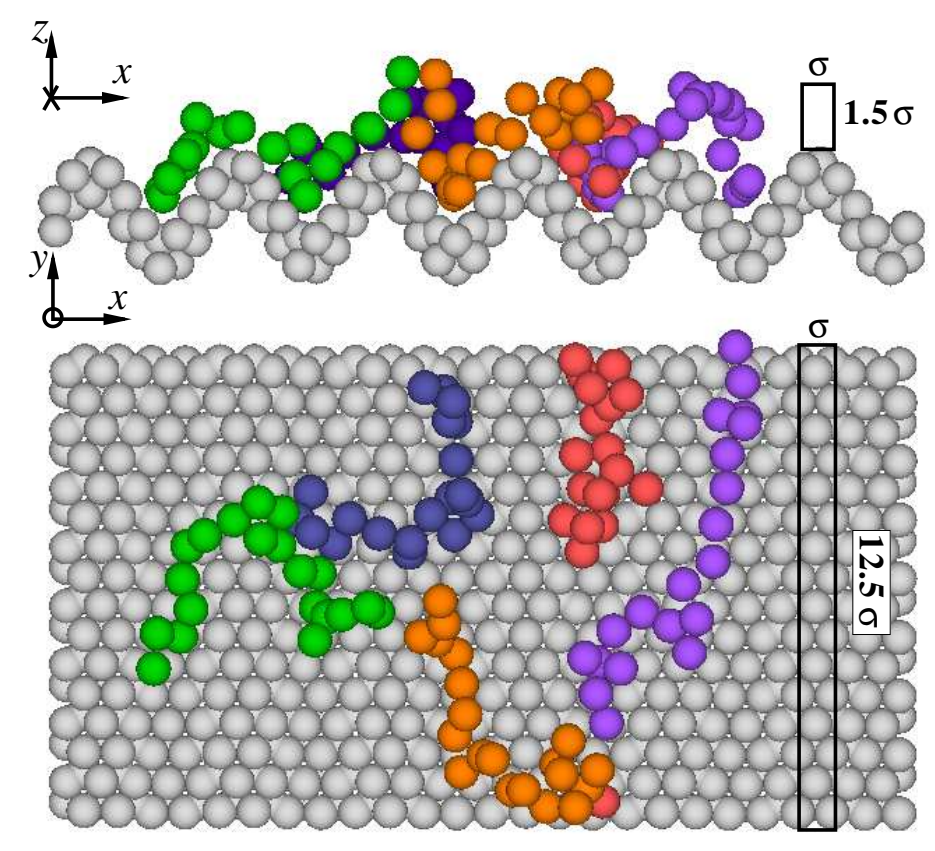

FIG. 11: (Color online) A snapshot of five polymer chains near the lower corrugated wall for the wavelength $\lambda=3.75 \sigma$ and amplitude $a=1.0 \sigma$. The figure shows the side view (top) and the top view (bottom). The velocity of the upper wall is $U=0.5 \sigma / \tau$.

are negligible. When the corrugation wavelengths are comparable to the radius of gyration, polymer chains stretch in the direction of shear flow above the crests of the surface corrugation, while the chains located in the grooves elongate perpendicular to the flow. In this regime the continuum approach fails to describe accurately the rapid decay of the effective slip length with increasing wavenumber.

\section{Acknowledgments}

Financial support from the Michigan State University Intramural Research Grants Program is gratefully acknowledged. The molecular dynamics simulations were conducted with the LAMMPS numerical code [64]. Computational work in support of this research was performed at Michigan State University's High Performance Computing Facility.

[1] A. A. Darhuber and S. M. Troian, Annu. Rev. Fluid Mech. 37, 425 (2005). 
[2] C. Neto, D. R. Evans, E. Bonaccurso, H. J. Butt, and V. S. J. Craig, Rep. Prog. Phys. 68, $2859(2005)$.

[3] N. V. Churaev, V. D. Sobolev, and A. N. Somov, J. Colloid Interface Sci. 97, 574 (1984).

[4] C. Cottin-Bizonne, S. Jurine, J. Baudry, J. Crassous, F. Restagno, and É. Charlaix, Eur. Phys. J. E 9, 47 (2002).

[5] L. Joly, C. Ybert, and L. Bocquet, Phys. Rev. Lett. 96, 046101 (2006).

[6] T. Schmatko, H. Hervet, and L. Leger, Phys. Rev. Lett. 94, 244501 (2005).

[7] R. Pit, H. Hervet, and L. Leger, Phys. Rev. Lett. 85, 980 (2000).

[8] Y. Zhu and S. Granick, Phys. Rev. Lett. 88, 106102 (2002).

[9] E. Bonaccurso, H. J. Butt, and V. S. J. Craig, Phys. Rev. Lett. 90, 144501 (2003).

[10] J. Sanchez-Reyes and L. A. Archer, Langmuir 19, 3304 (2003).

[11] T. Schmatko, H. Hervet, and L. Leger, Langmuir 22, 6843 (2006).

[12] O. I. Vinogradova and G. E. Yakubov, Phys. Rev. E 73, 045302(R) (2006).

[13] K. B. Migler, H. Hervet, and L. Leger, Phys. Rev. Lett. 70, 287 (1993).

[14] R. G. Horn, O. I. Vinogradova, M. E. Mackay, and N. Phan-Thien, J. Chem. Phys. 112, 6424 (2000).

[15] Y. Zhu and S. Granick, Phys. Rev. Lett. 87, 096105 (2001).

[16] V. S. J. Craig, C. Neto, and D. R. M. Williams, Phys. Rev. Lett. 87, 054504 (2001).

[17] C. H. Choi, K. J. A. Westin, and K. S. Breuer, Phys. Fluids 15, 2897 (2003).

[18] J. Koplik, J. R. Banavar, and J. F. Willemsen, Phys. Fluids A 1, 781 (1989).

[19] P. A. Thompson and M. O. Robbins, Phys. Rev. A 41, 6830 (1990).

[20] P. A. Thompson, M. O. Robbins, and G. S. Grest, Israel Journal of Chemistry 35, 93 (1995).

[21] P. A. Thompson and S. M. Troian, Nature (London) 389, 360 (1997).

[22] A. Jabbarzadeh, J. D. Atkinson, and R. I. Tanner, J. Chem. Phys. 110, 2612 (1999).

[23] J.-L. Barrat and L. Bocquet, Phys. Rev. Lett. 82, 4671 (1999).

[24] M. Cieplak, J. Koplik, J. R. Banavar, Phys. Rev. Lett. 86, 803 (2001).

[25] N. V. Priezjev and S. M. Troian, Phys. Rev. Lett. 92, 018302 (2004).

[26] T. M. Galea and P. Attard, Langmuir 20, 3477 (2004).

[27] N. V. Priezjev, Phys. Rev. E 75, 051605 (2007).

[28] R. Khare, J. J. de Pablo, and A. Yethiraj, Macromolecules 29, 7910 (1996).

[29] A. Koike and M. Yoneya, J. Phys. Chem. B 102, 3669 (1998). 
[30] A. Niavarani and N. V. Priezjev, Phys. Rev. E 77, 041606 (2008).

[31] J. P. Gao, W. D. Luedtke, and U. Landman, Tribol. Lett. 9, 3 (2000).

[32] A. Jabbarzadeh, J. D. Atkinson, and R. I. Tanner, Phys. Rev. E 61, 690 (2000).

[33] N. V. Priezjev and S. M. Troian, J. Fluid Mech. 554, 25 (2006).

[34] C. Kunert and J. Harting, Phys. Rev. Lett. 99, 176001 (2007).

[35] N. V. Priezjev, J. Chem. Phys. 127, 144708 (2007).

[36] E. Lauga and H. A. Stone, J. Fluid Mech. 489, 55 (2003).

[37] S. C. Hendy, M. Jasperse, and J. Burnell, Phys. Rev. E 72, 016303 (2005).

[38] N. V. Priezjev, A. A. Darhuber, and S. M. Troian, Phys. Rev. E 71, 041608 (2005).

[39] T. Qian, X. P. Wang, and P. Sheng, Phys. Rev. E 72, 022501 (2005).

[40] S. C. Hendy and N. J. Lund, Phys. Rev. E 76, 066313 (2007).

[41] S. Richardson, J. Fluid Mech. 59, 707 (1973).

[42] C. Cottin-Bizonne, J.-L. Barrat, L. Bocquet, and É. Charlaix, Nature Mat. 2, 237 (2003).

[43] C. Cottin-Bizonne, C. Barentin, É. Charlaix, L. Bocquet, and J.-L. Barrat, Eur. Phys. J. E 15, 427 (2004).

[44] M. Sbragaglia, R. Benzi, L. Biferale, S. Succi, and F. Toschi, Phys. Rev. Lett. 97, 204503 (2006).

[45] M. Sbragaglia and A. Prosperetti, Phys. Fluids 19, 043603 (2007).

[46] J. Ou, B. Perot, and J. P. Rothstein, Phys. Fluids 16, 4635 (2004).

[47] C. H. Choi and C. J. Kim, Phys. Rev. Lett. 96, 066001 (2006).

[48] P. Joseph, C. Cottin-Bizonne, J.-M. Benoit, C. Ybert, C. Journet, P. Tabeling, and L. Bocquet, Phys. Rev. Lett. 97, 156104 (2006).

[49] D. Einzel, P. Panzer, and M. Liu, Phys. Rev. Lett. 64, 2269 (1990).

[50] P. Panzer, M. Liu, and D. Einzel, Int. J. Mod. Phys. B 6, 3251 (1992)

[51] K. Kremer and G. S. Grest, J. Chem. Phys. 92, 5057 (1990).

[52] G. S. Grest and K. Kremer, Phys. Rev. A 33, 3628 (1986).

[53] J. P. Boon and S. Yip, Molecular Hydrodynamics (McGraw-Hill, New York, 1980).

[54] M. P. Allen and D. J. Tildesley, Computer Simulation of Liquids (Clarendon, Oxford, 1987).

[55] J. C. Heinrich and D. W. Pepper, Intermediate Finite Element Method: Fluid Flow and Heat Transfer Applications (Taylor and Francis, Philadelphia, 1999).

[56] J. H. Irving and J. G. Kirkwood, J. Chem. Phys. 18, 817 (1950). 
[57] R. B. Bird, C. F. Curtiss, R. C. Armstrong, and O. Hassager, Dynamics of Polymeric Liquids 2nd ed. (Wiley, New York, 1987).

[58] Z. Xu, J. J. de Pablo, and S. Kim, J. Chem. Phys. 102, 5836 (1995).

[59] J. T. Bosko, B. D. Todd, and R. J. Sadus, J. Chem. Phys. 121, 12050 (2004).

[60] L. M. Hocking, J. Fluid Mech. 76, 801 (1976).

[61] E. O. Tuck and A. Kouzoubov, J. Fluid Mech. 300, 59 (1995).

[62] T. Aoyagi, J. Takimoto, and M. Doi, J. Chem. Phys. 115, 552 (2001).

[63] I. Bitsanis and G. Hadziioannou, J. Chem. Phys. 92, 3827 (1990).

[64] S. J. Plimpton, J. Comp. Phys. 117, 1 (1995); see also URL http://lammps.sandia.gov. 